\title{
Effects of flow on the dynamics of a ferromagnetic nematic liquid crystal
}

\author{
Tilen Potisk ${ }^{1,2, *}$, Harald Pleiner ${ }^{3}$, Daniel Svenšek ${ }^{2}$, and Helmut R. Brand ${ }^{1}$ \\ ${ }^{1}$ Department of Physics, University of Bayreuth, 95440 Bayreuth, Germany \\ ${ }^{2}$ Department of Physics, Faculty of Mathematics and Physics, University of Ljubljana, SI-1000 Ljubljana, Slovenia \\ ${ }^{3}$ Max Planck Institute for Polymer Research, 55021 Mainz, Germany \\ *email: tilen.potisk@uni-bayreuth.de
}

(Dated: April 14, 2018)

\begin{abstract}
We investigate the effects of flow on the dynamics of ferromagnetic nematic liquid crystals. As a model we study the coupled dynamics of the magnetization, $\mathbf{M}$, the director field, $\mathbf{n}$, associated with the liquid crystalline orientational order and the velocity field $\mathbf{v}$. We evaluate how simple shear flow in a ferromagnetic nematic is modified in the presence of small external magnetic fields and we make experimentally testable predictions for the resulting effective shear viscosity: an increase by a factor of two in a magnetic field of about $20 \mathrm{mT}$. Flow alignment, a characteristic feature of classical uniaxial nematic liquid crystals, is analyzed for ferromagnetic nematics for the two cases of a magnetization in or perpendicular to the shear plane. In the former case we find that small in-plane magnetic fields are sufficient to suppress tumbling and that thus the boundary between flow alignment and tumbling can be controlled easily. In the latter case we furthermore find a possibility of flow alignment in a regime for which one obtains tumbling for the pure nematic component. We derive the analogues of the three Miesowicz viscosities well-known from usual nematic liquid crystals, corresponding to nine different configurations. Combinations of these can be used to determine several dynamic coefficients experimentally.
\end{abstract}

\section{INTRODUCTION}

Many complex fluids show anisotropic and/or nonNewtonian behavior in their flow properties. A class of anisotropic complex fluids for which the flow behavior has been studied in some detail because of their wideranging applications are liquid crystals, in particular nematic liquid crystals [1]. Uniaxial nematics are uniaxially anisotropic liquid systems which find applications, for example, in large area displays. Clearly the flow properties of the uniaxial nematic phase are the most studied and best understood among all liquid crystalline phases [1].

In parallel the field of magnetic liquids, i.e., suspensions of magnetic monodomain particles, has developed [2]. Various aspects of their characterization as well as of their macroscopic and microscopic properties are addressed in Refs. [3-8].

Quite recently there exists considerable interest in a novel type of nematic phase, namely ferromagnetic nematics, showing simultaneously nematic as well as ferromagnetic order. While such a phase has been predicted and investigated theoretically almost fifty years ago [9], only recently its synthesis has been reported experimentally $[10,11]$.

The ferromagnetic nematic is the first room temperature liquid multiferroic system. The only other liquid multiferroic systems known earlier are the superfluid phases of ${ }^{3} \mathrm{He}[12,13]$. Two of them, namely ${ }^{3} \mathrm{He}-\mathrm{A}$ and ${ }^{3} \mathrm{He}-\mathrm{A}_{1}$, are also uniaxial and show spontaneously broken orientational order as well as (anti)ferromagnetism. Besides superfluidity, both phases show rich macroscopic behavior including spin waves [14-18].

We also note that in the meantime ferromagnetic cholesteric phases have been described and characterized [19-21], thus complementing the abundant usual cholesteric phases, which break parity symmetry, because they are composed of chiral molecules: left and right handed helices differ from their mirror image in a nontrivial way, i.e., they cannot be brought to coincidence by mere rotations. The macroscopic behavior of ferrocholesterics has been elucidated in Ref. [22].

As for ferromagnetic nematics most studies focused on their synthesis, their characterization and their static properties [10, 11, 23]. There is also early work discussing a Landau description of phase transitions involving a ferromagnetic nematic phase [24]. Only quite recently the systematic investigation of their dynamic properties has started [25, 26]. These first two publications focused on the coupled dynamics of the two order parameters, namely the magnetization, $\mathbf{M}$, characterizing spontaneously broken rotational symmetry in spin space, and the director, $\mathbf{n}$, characteristic for systems with spontaneously broken rotational symmetry [27].

In this paper we analyze the coupling of these two order parameters to flows generalizing simple flow situations for uniaxial nematics to ferromagnetic nematics. For the new liquid multiferroic system, we include a discussion of the analogues of effective viscosity, Miesowicz viscosities, flow alignment as well as transient backflow [28, 29] and kickback [30] effects, all familiar from usual nematics [1]. The main goal of this study is to make concrete experimentally testable predictions.

The paper is organized as follows. In Sec. II we present the macroscopic model used throughout the present paper. In Sec. III we discuss simple shear flow and its experimentally accessible consequences. Sec. IV is dedicated to the characterization of the analogues of the Miesowicz viscosities for ferromagnetic nematic liquid crystals leading to many predictions. In Sec. $\mathrm{V}$ we consider the analogue of flow alignment and show how the transition 
to tumbling can be shifted by small external magnetic fields. In Sec. VI we sketch out the effects of flow on the the dynamic behavior when an external magnetic field is switched on. In the brief last Section we conclude and give a perspective.

\section{MACROSCOPIC MODEL}

Throughout the present paper we take into account the magnetization $\mathbf{M}$, the director field $\mathbf{n}$ and the velocity field $\mathbf{v}$ as macroscopic variables. For a complete set of macroscopic dynamic equations for ferronematics we refer to Refs. [31, 32].

The static behavior is described by the free energy density $f(\mathbf{M}, \mathbf{n}, \nabla \mathbf{n})$,

$f=-\mu_{0} \mathbf{M} \cdot \mathbf{H}-\frac{1}{2} A_{1}(\mathbf{M} \cdot \mathbf{n})^{2}+\frac{1}{2} A_{2}\left(|\mathbf{M}|-M_{0}\right)^{2}+f^{F}$,

where $\mu_{0}$ is the magnetic constant, $\mathbf{H}$ is the applied magnetic field, and $A_{1,2}>0$ will be assumed constant. The first term represents the coupling of the magnetization and the external magnetic field. The second term describes the static coupling between the director field and the magnetization (originating from the magnetic particles). The third term describes the energy connected with the deviation of the modulus of the magnetization from $M_{0}$. The last term is the Frank elastic energy associated with director distortions [1]

$$
\begin{aligned}
f^{F} & =\frac{1}{2} K_{1}(\nabla \cdot \mathbf{n})^{2}+\frac{1}{2} K_{2}[\mathbf{n} \cdot(\nabla \times \mathbf{n})]^{2} \\
& +\frac{1}{2} K_{3}[\mathbf{n} \times(\nabla \times \mathbf{n})]^{2},
\end{aligned}
$$

with positive elastic constants for splay $\left(K_{1}\right)$, twist $\left(K_{2}\right)$, and bend $\left(K_{3}\right)$. Throughout this paper, a one constant approximation will be used, i.e., $K_{1}=K_{2}=K_{3}=K$. While it is a good approximation to assume that $|\mathbf{M}|=$ $M_{0}$, we will take into account small variations of $|\mathbf{M}|$ (corresponding to large values of $A_{2}$ ).

The total free energy is $F=\int f \mathrm{~d} V$ and the equilibrium condition requires $\delta F=0$.

The macroscopic dynamic equations for the magnetization, the director field and the velocity field read [32, 33]

$$
\begin{aligned}
\left(\frac{\partial}{\partial t}+v_{j} \nabla_{j}\right) M_{i}+\epsilon_{i j k} M_{j} \omega_{k}+X_{i}^{R}+X_{i}^{D} & =0 \\
\left(\frac{\partial}{\partial t}+v_{j} \nabla_{j}\right) n_{i}+\epsilon_{i j k} n_{j} \omega_{k}+Y_{i}^{R}+Y_{i}^{D} & =0 \\
\rho\left(\frac{\partial}{\partial t}+v_{j} \nabla_{j}\right) v_{i}+\nabla_{j}\left(\sigma_{i j}^{R}+\sigma_{i j}^{D}+\sigma_{i j}^{t h}\right)-\nabla_{i} p & =0
\end{aligned}
$$

where $\omega_{i}=\frac{1}{2} \varepsilon_{i j k} \nabla_{j} v_{k}$ is the vorticity and $\rho$ is the density. The (quasi-)currents have been split into reversible $\left(X_{i}^{R}, Y_{i}^{R}, \sigma_{i j}^{R}\right)$ and irreversible, dissipative $\left(X_{i}^{D}, Y_{i}^{D}, \sigma_{i j}^{D}\right)$ parts. In Eq. (5) the reversible part of the stress tensor has been further split to include a thermodynamic part

$$
\sigma_{i j}^{t h}
$$

$$
\begin{aligned}
\sigma_{i j}^{t h} & =-B_{j} H_{i}-\frac{1}{2}\left(h_{i}^{n} n_{j}-h_{j}^{n} n_{i}\right) \\
& -\frac{1}{2}\left(h_{i}^{M} M_{j}-h_{j}^{M} M_{i}\right)+K_{k j m p} \nabla_{p} n_{m} \nabla_{i} n_{k},
\end{aligned}
$$

and the thermodynamic pressure

$$
p=-\varepsilon+T \sigma+\mu \rho+\mathbf{g} \cdot \mathbf{v}+\mathbf{B} \cdot \mathbf{H},
$$

with temperature $T$, entropy density $\sigma$, chemical potential $\mu$, density of linear momentum $\mathbf{g}$ and magnetic flux density B. The reversible (dissipative) parts of the (quasi-)currents have the same (opposite) behavior under time reversal as the time derivatives of the corresponding variables, i.e., Eqs. (3)-(5) are invariant under time reversal only if the dissipative (quasi-)currents vanish.

The (quasi-)currents are expressed as linear combinations of conjugate quantities (thermodynamic forces)

$$
\begin{aligned}
h_{i}^{M} & \equiv \frac{\delta f}{\delta M_{i}}=\frac{\partial f}{\partial M_{i}} \\
h_{i}^{n} & \equiv \delta_{i k}^{\perp} \frac{\delta f}{\delta n_{k}}=\delta_{i k}^{\perp}\left(\frac{\partial f}{\partial n_{k}}-\partial_{j} \Phi_{k j}\right) \\
A_{i j} & \equiv \frac{1}{2}\left(\partial_{i} v_{j}+\partial_{j} v_{i}\right)
\end{aligned}
$$

with $\Phi_{k j}=\partial f / \partial\left(\nabla_{j} n_{k}\right)$ and where the transverse Kronecker delta $\delta_{i k}^{\perp}=\delta_{i k}-n_{i} n_{k}$ projects onto the plane perpendicular to the director due to the constraint $\mathbf{n}^{2}=1$.

In Ref. [25] only the dissipative quasi-currents $X_{i}^{D}$ and $Y_{i}^{D}$ were taken into account as they had a direct relevance for the explanation of the experimental results discussed there. The effects of the reversible quasi-currents $X_{i}^{R}$ and $Y_{i}^{R}$ were modeled in Ref. [26]. In the present paper we also include the velocity variable in the approximation of an incompressible flow, $\nabla_{i} v_{i}=0$.

The dissipative quasi-currents take the form [32]

$$
\begin{aligned}
X_{i}^{D} & =b_{i j}^{D} h_{j}^{M}+\chi_{j i}^{D} h_{j}^{n}+c_{i j k}^{D} A_{j k}, \\
Y_{i}^{D} & =\frac{1}{\gamma_{1}} \delta_{i k}^{\perp} h_{k}^{n}+\chi_{i j}^{D} h_{j}^{M}+\lambda_{i j k}^{D} A_{j k}, \\
\sigma_{i j}^{D} & =-\nu_{i j k l}^{D} A_{k l}-\lambda_{k i j}^{D} h_{k}^{n}-c_{k i j}^{D} h_{k}^{M},
\end{aligned}
$$


with

$$
\begin{aligned}
\chi_{i j}^{D} & =\chi_{1}^{D} \delta_{i k}^{\perp} M_{k} n_{j}+\chi_{2}^{D} \delta_{i j}^{\perp} M_{k} n_{k} \\
b_{i j}^{D} & =b_{\|}^{D} n_{i} n_{j}+b_{\perp}^{D} \delta_{i j}^{\perp} \\
\nu_{i j k l}^{D} & =2\left(\nu_{1}+\nu_{2}-2 \nu_{3}\right) n_{i} n_{j} n_{k} n_{l} \\
& +\left(\nu_{3}-\nu_{2}\right)\left(n_{j} n_{l} \delta_{i k}+n_{j} n_{k} \delta_{i l}+n_{i} n_{k} \delta_{j l}+n_{i} n_{l} \delta_{j k}\right) \\
& +\left(\nu_{4}-\nu_{2}\right) \delta_{i j} \delta_{k l}+\nu_{2}\left(\delta_{j l} \delta_{i k}+\delta_{i l} \delta_{j k}\right) \\
& +\left(\nu_{5}-\nu_{4}+\nu_{2}\right)\left(\delta_{i j} n_{k} n_{l}+\delta_{k l} n_{i} n_{j}\right) \\
\lambda_{i j k}^{D} & =\lambda_{1}^{D}\left(\delta_{i q}^{\perp} \epsilon_{p j q} M_{p} n_{k}+\delta_{i q}^{\perp} \epsilon_{p k q} M_{p} n_{j}\right) \\
& +\lambda_{2}^{D}\left(\delta_{i k}^{\perp} \epsilon_{p j q} M_{p} n_{q}+\delta_{i j}^{\perp} \epsilon_{p k q} M_{p} n_{q}\right) \\
& +\lambda_{3}^{D}\left(\epsilon_{i p k} M_{j} n_{p}+\epsilon_{i p j} M_{k} n_{p}\right) \\
& +\lambda_{4}^{D} M_{q} n_{q}\left(\epsilon_{i p k} n_{j} n_{p}+\epsilon_{i p j} n_{k} n_{p}\right) \\
& +\lambda_{5}^{D} \epsilon_{p i q} M_{p} n_{q} n_{j} n_{k}+\lambda_{6}^{D} \epsilon_{p i q} M_{p} n_{q} \delta_{j k}^{\perp} \\
c_{i j k}^{D} & =c^{D}\left(\epsilon_{i m k} n_{m} n_{j}+\epsilon_{i m j} n_{m} n_{k}\right)
\end{aligned}
$$

The tensor $\chi_{i j}^{D}$ describes the dissipative cross-coupling between the director and the magnetization. In Refs. [25] and [26] it was shown that it is of major importance for the dynamics and was crucial to explain the presented experiments. The viscosity tensor $\nu_{i j k l}^{D}$ is the same as for usual nematic liquid crystals (in Appendix A the connection with the Leslie viscosity coefficients is reviewed). The existence of the magnetization gives rise to an additional dissipative coupling of the velocity with the director, described by the tensor $\lambda_{i j k}^{D}$, which is not present in usual nematics. Moreover, there exists also a direct dissipative coupling of the velocity and the magnetization, described by the tensor $c_{i j k}^{D}$. Throughout the present paper we will discard the biaxiality of the material which arises for $\mathbf{n} \nVdash \mathbf{M}$.

The reversible quasi-currents [32] are obtained by requiring that the entropy production $Y_{i} h_{i}^{n}+X_{i} h_{i}^{M}+\sigma_{i j} A_{j i}$ is zero. They are

$$
\begin{aligned}
X_{i}^{R} & =b_{i j}^{R} h_{j}^{M}+\chi^{R} \epsilon_{i j k} n_{j} h_{k}^{n}-c_{i j k}^{R} A_{j k} \\
Y_{i}^{R} & =\left(\gamma_{1}^{-1}\right)_{i j}^{R} h_{j}^{n}+\chi^{R} \epsilon_{i j k} n_{j} h_{k}^{M}-\frac{1}{2} \lambda_{i j k} A_{j k}, \\
\sigma_{i j}^{R} & =-\nu_{i j k l}^{R} A_{k l}-\frac{1}{2} \lambda_{k j i} h_{k}^{n}-c_{k i j}^{R} h_{k}^{M},
\end{aligned}
$$

In Eqs. (20)-(21), the reversible couplings between the velocity field on the one hand and the director field and the magnetization are described by the flow alignment tensor

$$
\lambda_{i j k}=\lambda\left(\delta_{i j}^{\perp} n_{k}+\delta_{i k}^{\perp} n_{j}\right) .
$$

and by the tensor $c_{i j k}^{R}[32]$, respectively,

$$
\begin{aligned}
c_{i j k}^{R} & =c_{1}^{R} M_{i} n_{j} n_{k}+c_{2}^{R}\left(\delta_{i j} M_{k}+\delta_{i k} M_{j}\right)+c_{3}^{R} M_{i} \delta_{j k} \\
& +c_{4}^{R} n_{i} M_{p} n_{p} \delta_{j k}+c_{5}^{R}\left(n_{i} M_{j} n_{k}+n_{i} M_{k} n_{j}\right) \\
& +c_{6}^{R} n_{i} M_{p} n_{p} n_{j} n_{k} .
\end{aligned}
$$

In the following we will discard the contributions of the tensors $\nu_{i j k l}^{R},\left(\gamma_{1}^{-1}\right)_{i j}^{R}, b_{i j}^{R}$ and $\chi^{R}$, which can be found in Ref. [32].

\section{A. Geometry and method}

In this paper we study the situation, where a ferromagnetic nematic liquid crystal is confined between a pair of infinite parallel plates separated in $z$ direction by the cell thickness $d$. We assume that the fields are functions only of the coordinate $z$ and time $t$. For solving Eqs. (3)-(5) a simple numerical method is used. We first discretize space into slices of width $\Delta z=d /(N-1)$, where $N$ is the number of discretization points. By varying $N$, it is found that using $N=50$ is already sufficient. We use a variant of the so called staggered grid, Fig. 1, to avoid possible numerical instabilities. The velocity field is defined in the middle of the slices, while the stress tensor, the director field and the magnetization field are defined at the edges of the slices. The velocity field at these edges can be calculated simply by averaging the neighboring points,

$$
v_{i}(j \Delta z)=0.5\left\{v_{i}[(j-1 / 2) \Delta z]+v_{i}[(j+1 / 2) \Delta z]\right\},
$$

where $\Delta z$ is the step size and $j \in\{0,1, \ldots, N-1\}$ is an integer.

After discretizing space one obtains $N$ ordinary differential equations. For the second derivative in the bulk of the liquid crystal cell we used the usual central finite difference scheme

$$
f^{\prime \prime}(z) \approx \frac{f(z+\Delta z)-2 f(z)+f(z-\Delta z)}{(\Delta z)^{2}}+\mathcal{O}\left((\Delta z)^{2}\right) .
$$

At the boundaries we use an asymmetric finite difference scheme for the derivatives.

Due to its simplicity we use the Euler method for our analysis. An example for one step of the Euler method for the i-th component of the director field at coordinate $\mathrm{z}$ is:

$$
n_{i}(t+\delta t, z)=n_{i}(t, z)-\delta t Y_{i}(t, z)+\mathcal{O}\left(\delta t^{2}\right),
$$

where $\delta t$ is the time step. An analogous equation holds for the magnetization field and the equations are solved simultaneously. Since the numerical scheme for the director field is not norm preserving, we normalize the director field after each time step: $n_{i} \rightarrow n_{i} /\left(n_{j} n_{j}\right)^{-1 / 2}$.

The velocity field relaxes on time scales much shorter than the director field or the magnetization and thus Eq. (5) can be simplified to

$$
\nabla_{j} \sigma_{i j}^{R}+\nabla_{j} \sigma_{i j}^{D}+\nabla_{j} \sigma_{i j}^{t h}-\nabla_{i} p=0 .
$$

The equation for the pressure field can be obtained by taking the divergence of Eq. (27). The boundary conditions for the pressure are obtained by taking the inner product of Eq. (27) with the surface normals, pointing up (down) at the top (bottom) plate. The resulting pressure field is

$$
p(z)=p_{0}+\sigma_{z z}(z),
$$


where $p_{0}$ is an arbitrary constant and $\sigma_{z z}$ includes all the stresses $\sigma_{z z}=\sigma_{z z}^{R}+\sigma_{z z}^{D}+\sigma_{z z}^{t h}$. Since $p$ is only a function of $z$ it only shows up in the dynamic equation for the $z$ component of the velocity field, where it exactly cancels out the contributions of all the stresses. The $z$ component of the velocity field is therefore independent of time, i.e., $\partial v_{z} / \partial t=0$, and is zero, $v_{z}=0$, due to the boundary condition.

Given the director and the magnetization field we solve Eq. (27) on a staggered grid for the velocity field, as shown in Fig. 1.

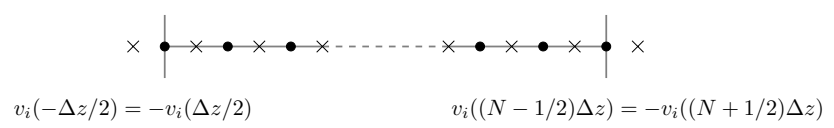

FIG. 1: A schematic representation of the staggered grid used to solve Eqs. (3)-(5). The director field, the magnetization field and the (quasi-)currents are defined on the points (black circles), while the velocity field is defined on the crosses located between the points. There are two crosses outside of the physical space, which are present in order to satisfy the boundary condition for the velocity field.

For the director field we assume infinitely strong (planar) anchoring at the boundaries, $\mathbf{n}=\hat{\mathbf{e}}_{x}$ at $z=0$ and $z=d$. For the velocity field we assume the no-slip condition $\mathbf{v}=0$ at $z=0$ and $z=d$. In the case of shear experiments, $\mathbf{v}=v_{0 x} \hat{\mathbf{e}}_{x}$ at $z=d$, where $v_{0 x}$ is the velocity of the upper plate. The lower plate is fixed. We define the shear rate

$$
\Gamma_{x}=\frac{v_{0 x}}{d}
$$

\section{B. Material parameters}

Throughout this study we will be using similar values of the static and the dynamic coefficients as determined in Ref. [25]. Therein a ferromagnetic nematic using 5CB as a nematic solvent was studied. For the static coefficients we used $A_{1}=130 \mu_{0}, A_{2}=1000 A_{1}, K=7 \mathrm{pN}$, $M_{0}=50 \mathrm{~A} / \mathrm{m}$ and $d=50 \mu \mathrm{m}$ and for the dynamic ones we used $\chi_{2}^{D}=20(\mathrm{Pas})^{-1}, \chi_{1}^{D}=0$, and $b_{\perp}^{D}=b_{\|}^{D}=$ $1.5 \times 10^{5} \mathrm{Am} / \mathrm{Vs}^{2}$. For the coefficients of the viscosity tensor, the rotational viscosity $\gamma_{1}$ and the flow alignment parameter $\lambda$ we use $\nu_{1}=0.092 \mathrm{Pas}, \nu_{2}=0.038 \mathrm{Pas}$, $\nu_{3}=0.045 \mathrm{Pas}, \gamma_{1}=0.081 \mathrm{Pas}$, and $\lambda=1.05$, see the data for 5CB in Ref. [34]. For the remaining coefficients of $\nu_{i j k l}^{D}$ we chose $\nu_{4}=\nu_{2}$ and $\nu_{5}=0$. In the literature one often encounters the so called Ericksen-Leslie viscosities, which are related to the set of viscosities used here (compare Appendix A for the detailed relations). For the reversible coupling $c_{i j k}^{R}$ between the magnetization and the velocity field in Eqs. (19) and (21), which is the analogue of the flow alignment tensor $\lambda_{i j k}$ of Eqs. (20)-(21), we use the coefficient $c_{2}^{R}$, Eq. (23), as a representative, since it has the same structure as $\lambda$ in Eq. (22). We choose the value $c_{2}^{R}=0.55$ so that its effects on the magnetization are comparable to the effects of $\lambda$ on the director field. It should be noted that for simple shear flow the contributions of $c_{3}^{R}$ and $c_{4}^{R}$ are automatically zero. Furthermore, due to the approximately fixed modulus of the magnetization, the contribution of the coefficient $c_{1}^{R}$ is negligible. The effect of $c_{5}^{R}$ is biggest when the magnetization (director) is parallel to the velocity field and the director (magnetization) is perpendicular to the velocity field and within the shear plane, which is perpendicular to the vorticity. Lastly, the effect of $c_{6}^{R}$ is biggest when the magnetization is either parallel or perpendicular to the velocity field and the director is at $45^{\circ}$ with respect to the magnetization while both fields are in the shear plane.

\section{SIMPLE SHEAR}

A classical approach to study the rheology of simple and complex fluids is the investigation of a simple shear flow. Here we will focus on the changes compared to the case of uniaxial nematics by the application of a magnetic field perpendicular to the plates of a shear cell.

In this section we discard the dissipative couplings of the director and the magnetization to the velocity field, i.e., the tensors $\lambda_{i j k}^{D}$ and $c_{i j k}^{D}$ of Eqs. (17) and (18) are put to zero. From the form of the tensor $\lambda_{i j k}^{D}\left(c_{i j k}^{D}\right)$ one can see that there is, in general, a nonzero coupling between the out-of-shear plane component of the director (magnetization) field with the part of the director (magnetization) molecular field $h_{i}^{n}\left(h_{i}^{M}\right)$, which is within the shear plane. Since we have put these tensors to zero, the velocity points along the $x$ axis everywhere with the director and the magnetization being within the shear plane, which is also confirmed numerically.

In Fig. 2 we present the solutions of $v_{x} / v_{0 x}, n_{z}$, and $M_{z} / M_{0}$ as functions of $z$. A magnetic field $\mathbf{H}=H \hat{\mathbf{e}}_{z}$ of $H=10 \mathrm{mT}$ Fig. 2(a) and $H=-10 \mathrm{mT}$ Fig. 2(b) was applied perpendicularly to the plates and a shear rate of $\Gamma_{x}=1 \mathrm{~s}^{-1}$ was imposed. We observe that for the negative magnetic field the director in the middle of the cell rotates by an angle of more than $\pi / 2$. This is due to the fact that the shear forces, which are described by the tensor $\lambda_{i j k}$, change direction at a certain orientation of the director. This orientation is determined by the parameter $\lambda$. Secondly, it is more favorable for the director to rotate further, since the coupling energy $\left(A_{1}\right)$ gets lower.

The dependence of the $x$ component of the velocity field on the magnetic field at a shear rate of $1 \mathrm{~s}^{-1}$ is shown in Fig. 3. One can see, as expected, that the velocity profile is not linear and a boundary layer of order $10 \%$ of the cell thickness is visible using $\Gamma_{x}=1 \mathrm{~s}^{-1}$ and magnetic fields of order $1 \mathrm{mT}$. The thickness of the boundary layer of the velocity field can be connected with the deformation of the director field, where the boundary layer is determined by the competition of the forces related to 

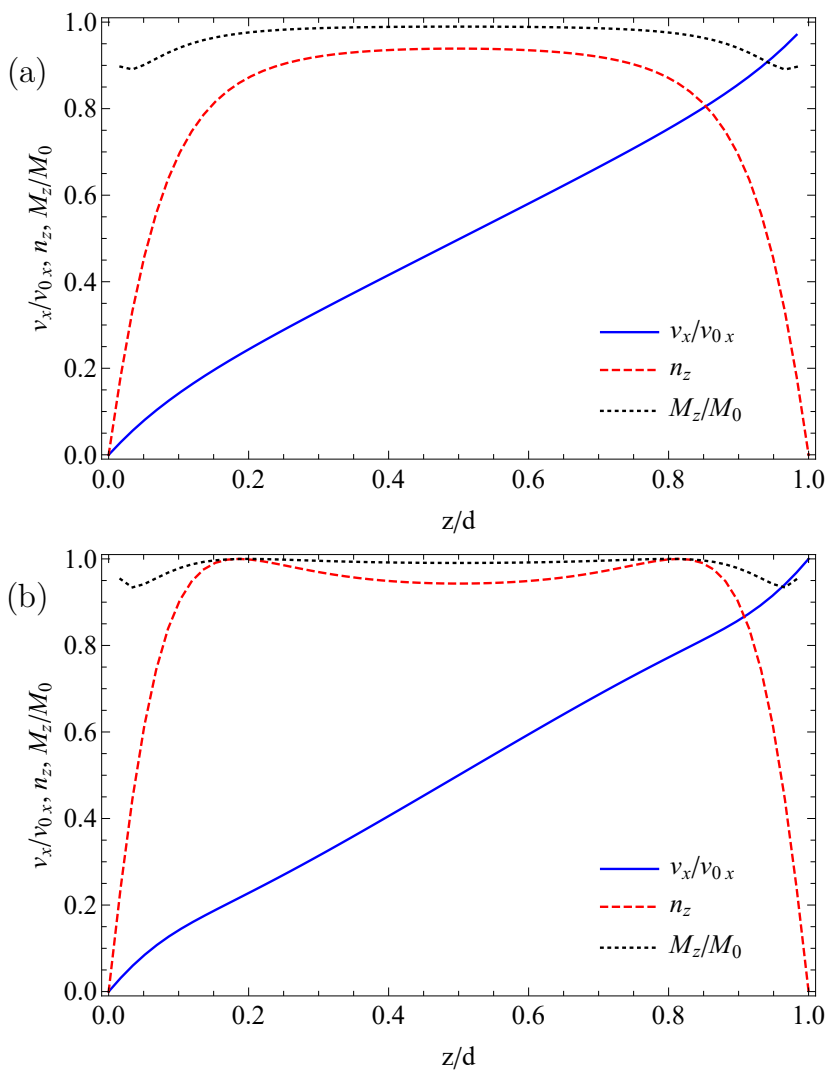

FIG. 2: Profiles of $v_{x} / v_{0 x}, M_{z} / M_{0}$ and $n_{z}$ at a shear rate $\Gamma_{x}=1 \mathrm{~s}^{-1}$ with (a) $\mu_{0} H=10 \mathrm{mT}$ and (b) $\mu_{0} H=-10 \mathrm{mT}$.

the static coupling $A_{1}$ and the Frank elastic forces.



FIG. 3: The normalized $x$ component of the velocity field $v_{x} / v_{0 x}$ for different values of the applied magnetic field at a shear rate $\Gamma_{x}=1 \mathrm{~s}^{-1}$.

The quantity that is normally measured is the effective viscosity of the sheared sample, i.e., the shear force per unit area $\sigma_{x z}$ exerted by the fluid on the glass plate divided by the shear rate $\Gamma_{x}$. In the present system, one must however take into account that the Maxwell stress $-B_{j} H_{i}$, unlike all the other contributions to the stress tensor, does not end at the boundary of the fluid as the magnetic flux continues into the glass plate. Consequently, the Maxwell stress does not contribute to the force on the glass plate. This is best seen by calculating the force as the integral of $\sigma_{x z}$ over a pair of planes tightly enclosing the $z=d$ interface. In the resulting difference of shear stresses across the interface, $\left[\sigma_{x z}\right]$, all contributions to $\sigma_{x z}(z=d)$ are recovered as usually, except the term $-B_{j} H_{i}$ of Eq. (6) which cancels out. The effective viscosity is thus

$$
\nu^{e f f}=\frac{\left[\sigma_{x z}\right]}{\Gamma_{x}} .
$$

In Fig. 4 the effective viscosity is plotted as a function of the magnetic field for two values of the shear rate $\Gamma_{x}$, which are equal in magnitude, but opposite in sign.

Most strikingly a rather large increase in the effective viscosity by about a factor of two can be achieved by applying a rather small magnetic field of about $20 \mathrm{mT}$, see Fig. 4(a). The reason the effective viscosity increases at very low fields is due to the response of the magnetization and the director field in an external magnetic field, which was studied in detail in Ref. [26]. There it was shown analytically that the configuration of the system, i.e., the director and the magnetization field, saturates quickly above a characteristic magnetic field determined by the static coupling, $\mu_{0} H \sim A_{1} M_{0}$, which is for the parameters used here approximately $10 \mathrm{mT}$. The viscosity increases as $\mathbf{n}$ and $\mathbf{M}$ rotate towards getting perpendicular to the velocity field and lying within the shear plane. This opens the door to an easily accessible viscosity control for a nematic fluid system by using small magnetic fields.

In addition, we see that - just as the absolute value of the vertical component of the director - also the effective viscosity is invariant with respect to the transformation $\Gamma_{x} \rightarrow-\Gamma_{x}, \mu_{0} H \rightarrow-\mu_{0} H$.

As we increase the magnetic field we see that the effective viscosity first saturates at fairly low magnetic fields of order $30 \mathrm{mT}$, Fig. 4(a). A similar effect has been observed in experiments using $8 \mathrm{CB}$ [35]. There, a rather complex shear geometry was used, therefore the experimental results cannot be mapped in a straightforward manner onto the results presented here.

In should be noted that we take into account also the diamagnetic contribution, $-\frac{1}{2} \mu_{0} \chi_{a}(\mathbf{n} \cdot \mathbf{H})^{2}$, with a diamagnetic anisotropy, $\chi_{a}=5 \times 10^{-6}$. For small magnetic fields, Fig. 4(a), the effects of this term are very small, which is verified by comparing the numerical results with $\chi_{a}=0$. On the other hand, if we increase the magnetic field further, the viscosity starts to increase again, see Fig. 4(b). This is due to the diamagnetic anisotropy, which tries to align the director along the external magnetic field. The viscosity finally saturates at fields of order $1 \mathrm{~T}$, where the director field along with the magnetization field point approximately along the applied magnetic field. In the limit of large magnetic fields, the director and the magnetization both point along $z$ and, 

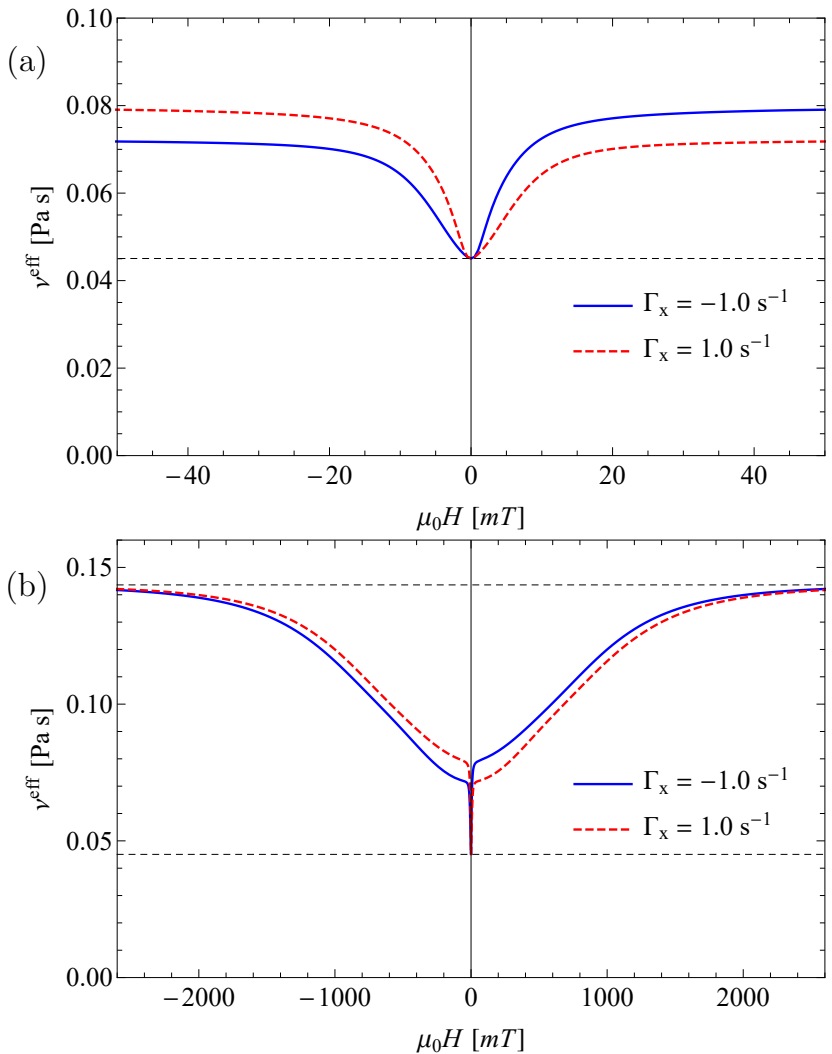

FIG. 4: The behavior of the effective viscosity for (a) small and (b) large values of the applied magnetic field at oppositely equal shear rates. The dashed lines represent two of the Miesowicz viscosities $\left(\eta_{x x}\right.$ and $\left.\eta_{z z}\right)$, defined in Sec. IV.

therefore, the effective viscosity converges to one of the Miesowicz viscosities, $\eta_{z z}$ (dashed line in Fig. 4(b)), defined and calculated in Sec. IV. In the absence of the magnetic field, the director and the magnetization point approximately along the $x$ axis, which means the effective viscosity approaches the value of another Miesowicz viscosity, $\eta_{x x}$, dashed line in Fig. 4(a). The behavior at larger magnetic fields is a prediction, which can be experimentally tested.

In Fig. 4 one also observes, that the effective viscosity strongly increases when one increases the magnetic field from the intermediate saturation region at $50 \mathrm{mT}$ to a large magnetic field of order of $1 \mathrm{~T}$. A possible explanation for such a dramatic increase can be deducted from Fig. 5, where we present the profiles of the fields $v_{x} / v_{0 x}$, $M_{z} / M_{0}$, and $n_{z}$ at these two magnetic fields. For the lower magnetic field Fig. 5(a), the boundary layer $\xi^{l}$ of $n_{z}$ is of order of 10 percent of the cell thickness, which can be estimated from the parameter $q$ of Eq. (39), discussed in the next subsection:

$$
\frac{\xi^{l}}{d} \sim \sqrt{\frac{K}{A_{1} M_{0}^{2} d^{2}}} \approx 0.1 .
$$

In the large magnetic field limit, the diamagnetic energy term is dominant. Equating the typical Frank elastic energy with the typical diamagnetic energy gives us a boundary layer $\xi^{q}$ of 2 percent of the cell thickness:

$$
\frac{\xi^{q}}{d} \sim \sqrt{\frac{K}{\mu_{0} \chi_{a} H^{2} d^{2}}} \approx 0.02 .
$$

Smaller boundary layers for the director field mean stronger elastic forces close to the boundaries. These forces increase the shear stress and therefore also the effective viscosity.
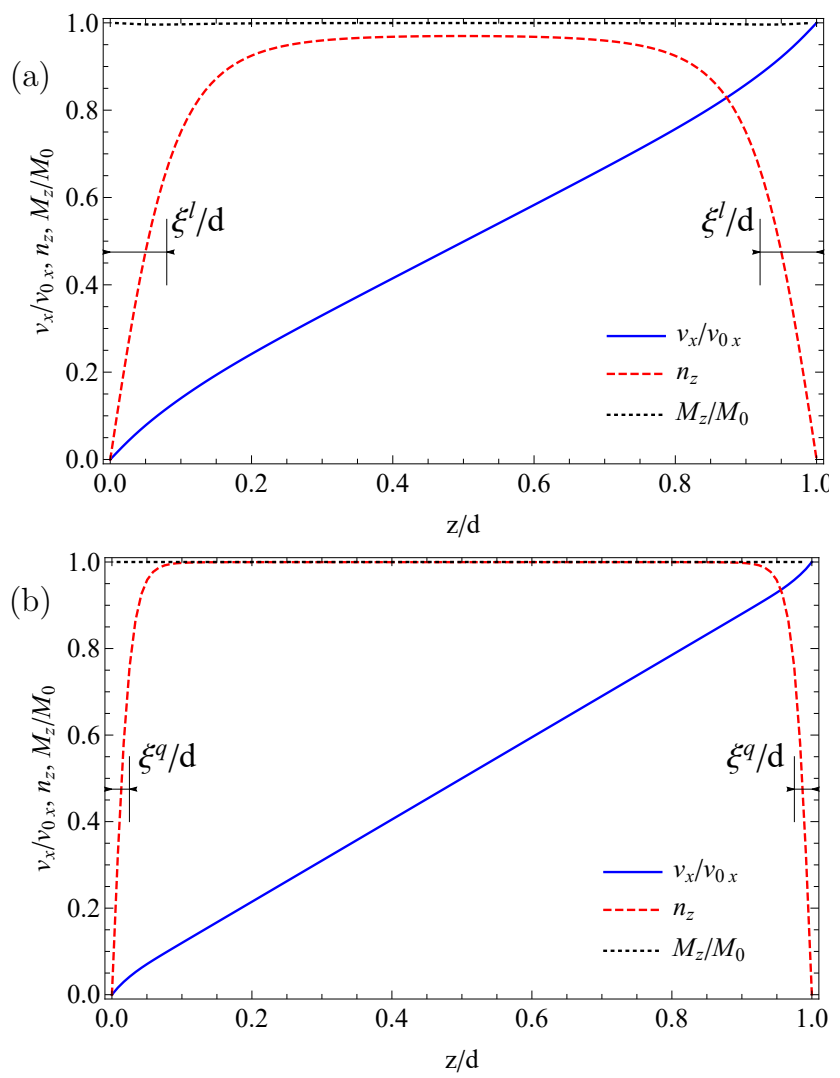

FIG. 5: Profiles of $v_{x} / v_{0 x}, M_{z} / M_{0}$, and $n_{z}$ at a shear rate $\Gamma_{x}=1 \mathrm{~s}^{-1}$ with (a) $\mu_{0} H=50 \mathrm{mT}$ and (b) $\mu_{0} H=1 \mathrm{~T}$. The boundary layers for (a) the lower magnetic field, $\xi^{l}$, and for (b) the higher magnetic field, $\xi^{q}$, can be estimated from Eqs. (31) and (32), respectively.

It should be noted that a crossover from a ferromagnetic response linear in $\mathrm{H}$, for small fields, to a regime quadratic in $\mathrm{H}$, for large fields, has also been observed in uniaxial magnetic gels [36], a class of soft matter systems which also shows rich macroscopic behavior [37].

\section{A. Small shear rate behavior}

For small shear rates $n_{z}$ increases linearly as we increase the shear rate. This can be shown analytically by first assuming that the velocity profile is linear, i.e. $\mathbf{v}=\Gamma_{x} z \hat{\mathbf{e}}_{x}$. We furthermore discard the dissipative cross-couplings 
between the velocity field and the director or the magnetization, described by the tensors $\lambda_{i j k}^{D}$ and $c_{i j k}^{D}$, Eqs. (17) and (18). Consequently, there are no terms in the dynamic equations Eqs. (4) and (3), which couple the thermodynamic forces to the currents in $y$ direction. The fields thus stay within the shear $(x z)$ plane,

$$
\begin{aligned}
\mathbf{n} & =\cos \theta \hat{\mathbf{e}}_{x}+\sin \theta \hat{\mathbf{e}}_{z}, \\
\mathbf{M} / M_{0} & =\cos \psi \hat{\mathbf{e}}_{x}+\sin \psi \hat{\mathbf{e}}_{z} .
\end{aligned}
$$

The dynamic equations for the angles $\theta$ and $\psi$ then read

$$
\begin{aligned}
\frac{\partial \theta}{\partial t} & =-\frac{1}{2} \Gamma_{x}[1-\lambda \cos (2 \theta)]+\frac{K}{\gamma_{1}} \frac{\partial^{2} \theta}{\partial z^{2}} \\
& -\frac{A_{1} M_{0}^{2}}{8}\left(\chi_{1}^{D}+\chi_{2}^{D}\right) \sin [4(\psi-\theta)] \\
& +\mu_{0} H M_{0}\left(\chi_{2}^{D} \cos ^{2}(\psi-\theta)-\chi_{1}^{D} \sin ^{2}(\psi-\theta)\right) \cos \psi \\
& +\frac{A_{1} M_{0}^{2}}{4}\left(\frac{2}{\gamma_{1}}+\chi_{1}^{D}-\chi_{2}^{D}\right) \sin [2(\psi-\theta)] \\
\frac{\partial \psi}{\partial t} & =-\frac{1}{2} \Gamma_{x}\left[1-2 c_{2}^{R} \cos (2 \psi)\right]+\frac{b_{\perp}^{D}}{M_{0}^{2}} \mu_{0} H M_{0} \cos \psi \\
& +\frac{1}{2}\left[\left(\chi_{1}^{D}+\chi_{2}^{D}\right) \cos [2(\psi-\theta)]-\chi_{1}^{D}+\chi_{2}^{D}\right] K \frac{\partial^{2} \theta}{\partial z^{2}} \\
& +\frac{A_{1} M_{0}^{2}}{8}\left(\chi_{1}^{D}+\chi_{2}^{D}\right) \sin [4(\psi-\theta)] \\
& -\frac{A_{1} M_{0}^{2}}{4}\left(\frac{2 b_{\perp}^{D}}{M_{0}^{2}}+\chi_{1}^{D}-\chi_{2}^{D}\right) \sin [2(\psi-\theta)] .
\end{aligned}
$$

In the small magnetic field limit we can use the small angle approximation $(\theta, \psi \ll 1)$. Setting the time derivatives $\partial \theta / \partial t$ and $\partial \psi / \partial t$ to zero, the solution for the angle $\theta$ reads:

$$
\begin{aligned}
& \theta(z)= \frac{\Gamma_{x}}{4 K\left(\frac{b_{\perp}^{D}}{\gamma_{1} M_{0}^{2}}-\left(\chi_{2}^{D}\right)^{2}\right)}\left[(\lambda-1)\left(\chi_{2}^{D}-\frac{b_{\perp}^{D}}{M_{0}^{2}}\right)\right. \\
&\left.-\left(2 c_{2}^{R}-1\right)\left(\frac{1}{\gamma_{1}}-\chi_{2}^{D}\right)\right] z(z-d) \\
&-\frac{\mu_{0} H M_{0}}{2 K} z(z-d)
\end{aligned}
$$

from which one can see that for small magnetic fields and shear rates $n_{z}$ is linear in both of them. In the limit $\Gamma_{x} \rightarrow 0$, one obtains a solution in agreement with the one in Ref. [26]. It is interesting to note that for small shear rates and in the absence of the magnetic field, the angle can decrease as one increases the shear rate, provided that the following inequality is satisfied:

$$
2 c_{2}^{R}>1+(\lambda-1) \frac{\chi_{2}^{D}-\frac{b_{\perp}^{D}}{M_{0}^{2}}}{\frac{1}{\gamma_{1}}-\chi_{2}^{D}} .
$$

It is rather realistic that this inequality is satisfied using known and/or extracted values of the involved parameters.
In the large magnetic field limit, the director and the magnetization point approximately along the $z$ axis. The solution for the angles $\theta^{+}\left(\theta^{-}\right)$for positive (negative) magnetic fields is

$$
\theta^{ \pm}(z)= \pm \frac{\pi}{2}-C \Gamma_{x} \mp\left(\frac{\pi}{2} \mp C \Gamma_{x}\right) \frac{\cosh [q(z-d / 2)]}{\cosh (q d / 2)},
$$

where

$$
q^{2}=q_{0}^{2} \frac{\mu_{0}|H| M_{0}}{A_{1} M_{0}^{2}+\mu_{0}|H| M_{0}},
$$

with $q_{0}=\sqrt{A_{1} M_{0}^{2} / K}$, and $C$ is a constant determined by the dynamic and the static parameters:

$$
\begin{aligned}
C & =\frac{\gamma_{1}(1+\lambda) b_{\perp}^{D}+\left(1-d_{2}\right) M_{0}^{2}}{2 \mu_{0}|H| M_{0}\left(b_{\perp}^{D}-\left[\chi_{2}^{D}\right]^{2} \gamma_{1} M_{0}^{2}\right)} \\
& +\frac{\gamma_{1}(1+\lambda) b_{\perp}^{D}-\left(1+2 c_{2}^{R}\right) \chi_{2}^{D} \gamma_{1} M_{0}^{2}}{2 A_{1} M_{0}^{2}\left(b_{\perp}^{D}-\left[\chi_{2}^{D}\right]^{2} \gamma_{1} M_{0}^{2}\right)}
\end{aligned}
$$

with the abbreviation $d_{2}=2 c_{2}^{R}\left(\chi_{2}^{D} \gamma_{1}-1\right)+\chi_{2}^{D} \gamma_{1}(2+\lambda)$. We point out that Eq. (38) correctly predicts the fact that the director can rotate by more than $\pi / 2$ in the middle of the cell, as is observed in Fig. 2.

\section{MIESOWICZ VISCOSITIES}

There exists a number of different ways one can measure viscosities of a nematic liquid crystal. The earliest technique and a particularly useful one is the concept of Miesowicz viscosities, where one fixes by an external magnetic or electric field the director and exposes the system to a shear flow $[38,39]$. Depending on the relative orientation of the director with respect to the velocity field or the shear plane, Fig. 6, there exist, for sufficiently high external fields, three limiting cases of the measured viscosities: $\eta_{x}$ when $\mathbf{n}=\hat{\mathbf{e}}_{x}, \eta_{y}$ when $\mathbf{n}=\hat{\mathbf{e}}_{y}$ and $\eta_{z}$ when $\mathbf{n}=\hat{\mathbf{e}}_{z}$. The shear flow is applied in the $x z$ plane. These Miesowicz viscosities are

$$
\begin{aligned}
& \eta_{x}=\nu_{3}+\frac{\gamma_{1}}{4}(\lambda-1)^{2}, \\
& \eta_{y}=\nu_{2}, \\
& \eta_{z}=\nu_{3}+\frac{\gamma_{1}}{4}(\lambda+1)^{2} .
\end{aligned}
$$

Below, we derive analogous viscosities for the ferromagnetic nematic liquid crystal. Since we have the additional variable of magnetization, not only are the expressions for the viscosities different but there are also more possible combinations. We will denote the analogous viscosities by $\eta_{\alpha \beta}$, where $\alpha, \beta \in\{x, y, z\}$ represent the fixed directions of the director and the magnetization, respectively. A simple shear flow $v_{x}(z)=\Gamma_{x} z, v_{y}(z)=$ $0, v_{z}(z)=0$ is imposed. The resulting nine independent possible configurations of the magnetization and the director are shown in Fig. 7 . 


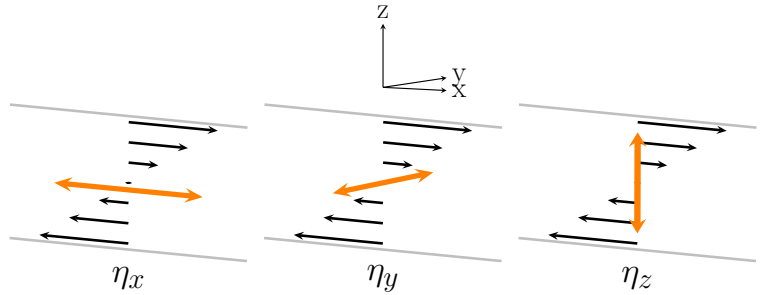

FIG. 6: Miesowicz viscosities in a usual nematic liquid crystal. The director field is indicated in orange.

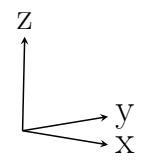

(a)

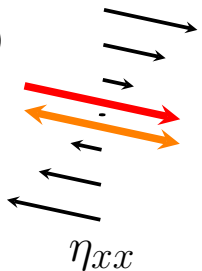

(b)



(c)




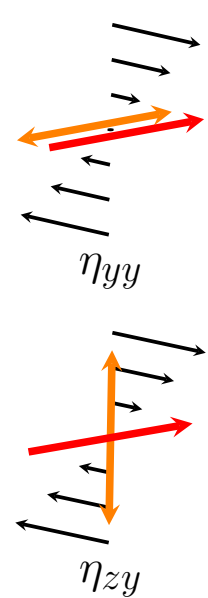
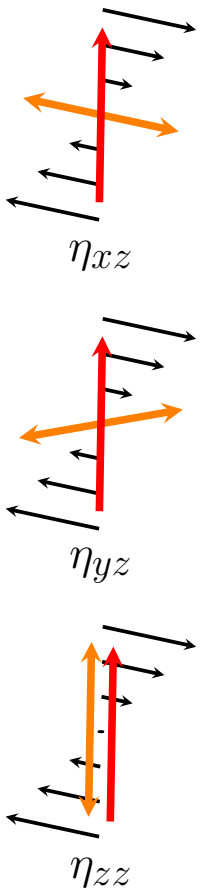

FIG. 7: Analogues of the Miesowicz viscosities in a ferromagnetic nematic liquid crystal, when the director (orange) is along the (a) $x$, (b) $y$ and (c) $z$ axis. The magnetization is shown in red.

To simplify the expressions, we have put, besides the tensors $\nu_{i j k l}^{R},\left(\gamma_{1}^{-1}\right)_{i j}^{R}, b_{i j}^{R}$ and $\chi^{R}$, also the tensor $\lambda_{i j k}^{D}$ and $\chi_{1}^{D}$ to zero, which is the same as in Sec. III, but here we keep $c_{i j k}^{D}$, Eq. (18). To derive the Miesowicz viscosities one first sets the quasi-currents $X_{i}$ and $Y_{i}$ to zero. From this one obtains the thermodynamic forces $h_{i}^{n}$ and $h_{i}^{M}$, Eqs. (9) and (8), as a function of the shear rate $\Gamma_{x}$. Finally, one uses the thermodynamic forces in the expression for the $x z$ component of the total stress tensor $\sigma_{x z}$.

When the director is along the $x$ axis, Fig. 7(a), the three independent viscosities are

$$
\begin{aligned}
\eta_{x x}= & \nu_{3}+\frac{1}{4}\left(1-\frac{\left(\chi_{2}^{D}\right)^{2} M_{0}^{2} \gamma_{1}}{b_{\perp}^{D}}\right)^{-1} \\
\times & {\left[\gamma_{1}(1-\lambda)^{2}-4\left(c^{D}\right)^{2}\right.} \\
& \left.+\frac{M_{0}^{2}}{b_{\perp}^{D}}\left(1-2 c_{2}^{R}\right)\left(1-2 c_{2}^{R}-2 \chi_{2}^{D} \gamma_{1}(1-\lambda)\right)\right] \\
\eta_{x y}= & \nu_{3}+\frac{\gamma_{1}}{4}(1-\lambda)^{2}-\frac{\left(c^{D}\right)^{2}}{b_{\perp}^{D}} \\
\eta_{x z}= & \nu_{3}+\frac{\gamma_{1}}{4}(1-\lambda)^{2}-\frac{\left(c^{D}\right)^{2}}{b_{\perp}^{D}} \\
& +\frac{M_{0}^{2}\left(1+2 c_{2}^{R}+2 c_{5}^{R}\right)^{2}}{4 b_{\|}^{D}}
\end{aligned}
$$

When the director is along the $y$ axis, Fig.7(b), the three independent viscosities are

$$
\begin{aligned}
& \eta_{y x}=\nu_{2}+\frac{M_{0}^{2}\left(1-2 c_{2}^{R}\right)^{2}}{4 b_{\perp}^{D}}, \\
& \eta_{y y}=\nu_{2}, \\
& \eta_{y z}=\nu_{2}+\frac{M_{0}^{2}\left(1+2 c_{2}^{R}\right)^{2}}{4 b_{\perp}^{D}} .
\end{aligned}
$$

One can see that in the case where the director and the magnetization are both perpendicular to the shear plane, the viscosity is $\eta_{y y}=\eta_{y}=\nu_{2}$ like in ordinary nematic liquid crystals.

When the director is along the $z$ axis, Fig.7(c), the viscosities are similar as in the case where the director is along the $x$ axis. One can get the viscosities $\eta_{z i}$ from $\eta_{x i}$ by the transformation $\lambda \rightarrow-\lambda, c_{2}^{R} \rightarrow-c_{2}^{R}, c_{5}^{R} \rightarrow-c_{5}^{R}$ and $x \leftrightarrow z$. This can be explained by the fact that the contributions of $\lambda, c_{2}^{R}$ and $c_{5}^{R}$ in the quasi-currents change sign as one rotates the director or the magnetization by $90^{\circ}$ within the shear plane. We thus have

$$
\begin{aligned}
\eta_{z x}= & \nu_{3}+\frac{\gamma_{1}}{4}(1+\lambda)^{2}-\frac{\left(c^{D}\right)^{2}}{b_{\perp}^{D}} \\
& +\frac{M_{0}^{2}\left(1-2 c_{2}^{R}-2 c_{5}^{R}\right)^{2}}{4 b_{\|}^{D}} \\
\eta_{z y}= & \nu_{3}+\frac{\gamma_{1}}{4}(1+\lambda)^{2}-\frac{\left(c^{D}\right)^{2}}{b_{\perp}^{D}} \\
\eta_{z z}= & \nu_{3}+\frac{1}{4}\left(1-\frac{\left(\chi_{2}^{D}\right)^{2} M_{0}^{2} \gamma_{1}}{b_{\perp}^{D}}\right)^{-1} \\
\times & {\left[\gamma_{1}(1+\lambda)^{2}-4\left(c^{D}\right)^{2}\right.} \\
& \left.+\frac{M_{0}^{2}}{b_{\perp}^{D}}\left(1+2 c_{2}^{R}\right)\left(1+2 c_{2}^{R}-2 \chi_{2}^{D} \gamma_{1}(1+\lambda)\right)\right] .
\end{aligned}
$$

We observe, that the viscosities reduce to Miesowicz viscosities, Eqs. (41)-(43), in the limit $c^{D} \rightarrow 0$ and $M_{0} \rightarrow 0$. 
In Refs. [25, 26] the value of the dissipative crosscoupling coefficient $\chi_{2}^{D}$ was shown to be large and thus it should strongly affect the values of the Miesowicz viscosities $\eta_{x x}$ and $\eta_{z z}$ as compared with the nematic analogues $\eta_{x}$ and $\eta_{y}$.

One notices that the Miesowicz viscosities only contain the coefficients $c_{2}^{R}$ and $c_{5}^{R}$ of $c_{i j k}^{R}$. As discussed in Sec. III, for simple shear the coefficients $c_{3}^{R}$ and $c_{4}^{R}$ do not contribute. The coefficient $c_{1}^{R}$ is irrelevant due to the fixed modulus $M_{0}$. The contributions of $c_{6}^{R}$ are zero in the chosen configurations, which is due to either the fixed modulus or the perpendicular orientations of the director and the magnetization.

We emphasize that important relations between the nine Miesowicz viscosities exist, e.g.,

$$
\begin{aligned}
\eta_{y z}-\eta_{y x} & =\frac{M_{0}^{2} c_{2}^{R}}{b_{\perp}^{D}} \\
\eta_{z z}-\eta_{x x} & =\frac{b_{\perp}^{D} \gamma_{1} \lambda-\left(2 c_{2}^{R}\left(\chi_{2}^{D} \gamma_{1}-1\right)+\chi_{2}^{D} \gamma_{1} \lambda\right) M_{0}^{2}}{b_{\perp}^{D}-\left(\chi_{2}^{D}\right)^{2} M_{0}^{2} \gamma_{1}} \\
\eta_{z x}-\eta_{x z} & =\gamma_{1} \lambda-\frac{M_{0}^{2}\left(c_{2}^{R}+c_{5}^{R}\right)}{b_{\|}^{D}}
\end{aligned}
$$

which can be used to determine certain combinations of dynamic coefficients experimentally.

\section{FLOW ALIGNMENT}

In this section we study the flow alignment in a ferromagnetic nematic. For usual uniaxial nematics this is a well-known phenomenon, where under the influence of a simple shear flow the director is tilted by a finite angle with respect to the velocity field. In the case of uniaxial nematics this angle is determined by the flow alignment parameter $\lambda$, which is a reversible transport coefficient and not associated with any dissipation. For biaxial nematics and for mixtures of uniaxial nematics various aspects of flow alignment have also been addressed [4044]. In ferromagnetic nematic liquid crystals we have to take into account also the dynamics of the magnetization. This means that in simple shear flow, generally, the director and the magnetization are not parallel.

We investigate the case, where the shear flow is imposed with the external magnetic field pointing along the $x$ axis, $\mathbf{H}=H \hat{\mathbf{e}}_{x}$, as opposed to the case in Sec. III, where the magnetic field points in $z$ direction. This direction of the magnetic field is chosen to ensure that the director and the magnetization field are in the presence of a magnetic field homogeneous across the cell. The homogeneous response makes it convenient to analyze the ferromagnetic nematic flow alignment as a function of the applied magnetic field. The contribution of the Frank elastic term, Eq. (2), can be thus discarded. Generally there exists a boundary layer with thickness

$$
\xi^{v} \sim \sqrt{\frac{K}{\gamma_{1} \Gamma_{x}}},
$$

which decreases with increasing shear rate. This boundary layer is defined by a competition between the viscous forces and the elastic forces. In the limit $\xi^{v} / d \ll 1$, the velocity profile is linear, $\mathbf{v}=\Gamma_{x} z \hat{\mathbf{e}}_{x}$. For the parameters used in this study, this limit can be achieved using shear rates $\Gamma_{x} \gg 0.03 \mathrm{~s}^{-1}$. We remind the reader that two different boundary layers, corresponding to the deformation of the director field in a magnetic field, are defined in Sec. III, Eqs. (31) and (32). Since we discard the elastic forces, these boundary layers are zero.

As is done in Sec. III, we again discard the dissipative cross-couplings between the velocity field and the director or the magnetization, Eqs. (17) and (18). In this case the director and the magnetization both stay in the shear ( $x z$ ) plane and can be described by Eq. (33), as discussed in Sec. III.

The dynamic equations for the angles $\theta$ and $\psi$ are then similar to the ones presented in Sec. III, Eqs.(34) and (35), with the difference being in the term describing the magnetic field and in the absence of elastic forces:

$$
\begin{aligned}
\frac{\partial \theta}{\partial t}= & -\frac{1}{2} \Gamma_{x}[1-\lambda \cos (2 \theta)]-\frac{A_{1} M_{0}^{2}}{8} \chi_{2}^{D} \sin [4(\psi-\theta)] \\
& -\chi_{2}^{D} \mu_{0} H M_{0} \cos ^{2}(\psi-\theta) \sin (\psi) \\
& +\frac{A_{1} M_{0}^{2}}{4}\left(\frac{2}{\gamma_{1}}-\chi_{2}^{D}\right) \sin [2(\psi-\theta)] \\
\frac{\partial \psi}{\partial t}= & -\frac{1}{2} \Gamma_{x}\left[1-2 c_{2}^{R} \cos (2 \psi)\right]+\frac{A_{1} M_{0}^{2}}{8} \chi_{2}^{D} \sin [4(\psi-\theta)] \\
& -\frac{b_{\perp}^{D}}{M_{0}^{2}} \mu_{0} H M_{0} \sin (\psi) \\
& -\frac{A_{1} M_{0}^{2}}{4}\left(\frac{2 b_{\perp}^{D}}{M_{0}^{2}}-\chi_{2}^{D}\right) \sin [2(\psi-\theta)]
\end{aligned}
$$

where, for simplicity $c_{2}^{R}$ is taken as a representative of $c_{i j k}^{R}$ and $\chi_{1}^{D}$ is set to zero. A stationary solution exists if $\partial \theta / \partial t=0$ and $\partial \psi / \partial t=0$.

In the limit of large shear rates $\left(\Gamma_{x} \gg \frac{A_{1} M_{0}^{2}}{\gamma_{1}}\right)$ and in zero magnetic field we obtain

$$
\begin{aligned}
\cos (2 \theta) & =\frac{1}{\lambda}, \\
\cos (2 \psi) & =\frac{1}{2 c_{2}^{R}} .
\end{aligned}
$$

The solutions of Eqs. (59) and (60) are $\theta=$ $\pm \frac{1}{2} \arccos (1 / \lambda)$ and $\psi= \pm \frac{1}{2} \arccos \left(1 / 2 c_{2}^{R}\right)$. A linear stability analysis of Eqs. (57) and (58) has been made, where the angles are perturbed from their stationary values $\theta_{0}$ and $\psi_{0}$, i.e. $\theta=\theta_{0}+\delta \theta$ and $\psi=\psi_{0}+\delta \psi$. We find that for positive (negative) shear rates, the positive (negative) angle is the stable solution. 
In the large magnetic field limit, $\frac{b_{\perp}^{D}}{M_{0}^{2}} \mu_{0} H M_{0} \gg \frac{A_{1} M_{0}^{2}}{\gamma_{1}}$, while still assuming that the effects of the diamagnetic anisotropy are negligible, stationary solutions $\psi^{ \pm}$for the magnetization angle are

$$
\sin \psi^{ \pm}=-\frac{\Gamma_{x}^{H}}{\Gamma_{x}} \pm \sqrt{\left(\frac{\Gamma_{x}^{H}}{\Gamma_{x}}\right)^{2}+\frac{2 c_{2}^{R}-1}{4 c_{2}^{R}}}
$$

where

$$
\Gamma_{x}^{H}=\frac{b_{\perp}^{D}}{4 c_{2}^{R} M_{0}^{2}} \mu_{0} H M_{0}
$$

is the characteristic 'magnetic' shear rate determined by the magnetic field.

It has not been possible to make the analytical solution for the angle $\theta$ tractable. We thus only present the asymptotic behaviour of this angle and for completeness also the asymptotic behavior of the angle $\psi$. From Eq. (61) one can see that in the limit $\Gamma_{x} / \Gamma_{x}^{H} \ll 1$ one finds, to first order,

$$
\begin{aligned}
\psi^{+}= & \frac{2 c_{2}^{R}-1}{8 c_{2}^{R} \frac{\Gamma_{x}}{\Gamma_{x}^{H}},} \\
\tan \theta^{+}= & \sqrt{\frac{b_{\perp}^{D}(\lambda-1)-\left(2 c_{2}^{R}-1\right) \chi_{2}^{D} M_{0}^{2}}{b_{\perp}^{D}(1+\lambda)}} \\
& -\frac{\left(2 c_{2}^{R}-1\right)^{2} \chi_{2}^{D} M_{0}^{2}}{8 b_{\perp}^{D} c_{2}^{R}(1+\lambda)} \frac{\Gamma_{x}}{\Gamma_{x}^{H}},
\end{aligned}
$$

while $\psi^{-}$does not exist and $\tan \theta^{+}$is calculated by inserting the solution for the angle $\psi^{+}$from Eq. (61) into Eq. (57). It is tractable to perform a linear stability analysis of the solution Eq. (58) analytically, finding the stability condition

$$
\pm c_{2}^{R} \Gamma_{x} \sqrt{\left(\frac{\Gamma_{x}^{H}}{\Gamma_{x}}\right)^{2}+\frac{2 c_{2}^{R}-1}{4 c_{2}^{R}}}>0,
$$

where the sign \pm corresponds to the solutions $\psi^{ \pm}$. From Eq. (65) we see that the solution $\psi^{+}\left(\psi^{-}\right)$is stable if the product $c_{2}^{R} \Gamma_{x}$ is positive (negative).

In Fig. 8 we present the numerical solutions of Eqs. (57) and (58) for the angles $\psi^{+}$and $\theta^{+}$as functions of $\Gamma_{x}^{H} / \Gamma_{x}$. As predicted in Eqs. (63) and (64), we see that the angle $\psi^{+}$decreases to zero, while the angle $\theta^{+}$saturates at a finite value as the field is increased.

In the absence of the magnetic field and in the large shear rate limit, the stationary solution exists if $|\lambda| \geq 1$ and $\left|c_{2}^{R}\right| \geq \frac{1}{2}$. In usual nematic liquid crystals, if $|\lambda|<1$ holds, the system shows a tumbling behavior. In such a system flow alignment can be recovered if a sufficiently large electric field is applied, see Refs. [45, 46]. In our system this could be achieved with the use of low magnetic fields.

To check this possibility, one must first ensure the existence of the solutions Eq. (61). Secondly, we are only interested in the stable solutions, i.e., Eq. (65) must hold.

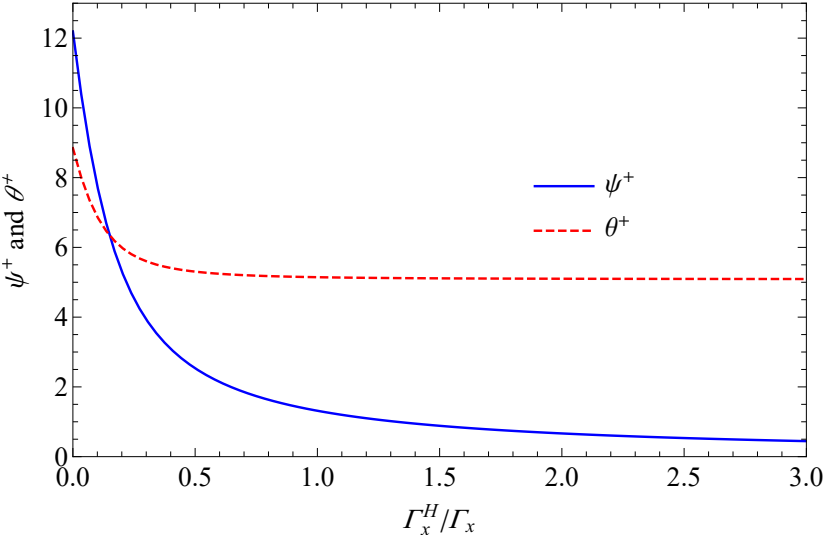

FIG. 8: The theoretical dependence of the magnetization $\psi^{+}$ (blue curve) and director $\theta^{+}$(red dashed curve) angles in degrees as functions of the dimensionless ratio of the characteristic magnetic shear rate Eq. (62) and the applied shear rate, $\Gamma_{x}^{H} / \Gamma_{x}$.

We find that the required magnetic field depends on four different ranges of the $c_{2}^{R}$ values:

$$
\begin{aligned}
& c_{2}^{R}<0, \quad \pm \Gamma_{x}<0, \quad \frac{\Gamma_{x}^{H}}{\Gamma_{x}}>\mp \frac{1+2 c_{2}^{R}}{8 c_{2}^{R}}, \\
& 0<c_{2}^{R}<\frac{1}{6}, \quad \pm \Gamma_{x}>0, \quad \frac{\Gamma_{x}^{H}}{\Gamma_{x}}> \pm \frac{1+2 c_{2}^{R}}{8 c_{2}^{R}}, \\
& \frac{1}{6}<c_{2}^{R}<\frac{1}{2}, \quad \pm \Gamma_{x}>0, \quad \frac{\Gamma_{x}^{H}}{\Gamma_{x}}> \pm \sqrt{\frac{1-2 c_{2}^{R}}{4 c_{2}^{R}}} \\
& c_{2}^{R}>\frac{1}{2}, \quad \pm \Gamma_{x}>0, \quad \frac{\Gamma_{x}^{H}}{\Gamma_{x}}>\mp \frac{1+2 c_{2}^{R}}{8 c_{2}^{R}},
\end{aligned}
$$

where the signs \pm correspond to the two solutions Eq. (61).

Another stationary solution of the dynamic equations is found when the director is in the shear plane, while the magnetization is perpendicular to this plane,

$$
\begin{aligned}
\mathbf{n} & =\cos \theta \hat{\mathbf{e}}_{x}+\sin \theta \hat{\mathbf{e}}_{z}, \\
\mathbf{M} & =M_{0} \hat{\mathbf{e}}_{y} .
\end{aligned}
$$

In this case we do not discard any dynamic coefficients. The solution for the angle $\theta$ is

$$
\tan (\theta)=\frac{\lambda_{\mathrm{eff}}^{D} M_{0}}{\lambda+1} \pm \sqrt{\left(\frac{\lambda_{\mathrm{eff}}^{D} M_{0}}{\lambda+1}\right)^{2}+\frac{\lambda-1}{\lambda+1}}
$$

where $\lambda_{\text {eff }}^{D}=2 \lambda_{1}^{D}+2 \lambda_{2}^{D}-\lambda_{5}^{D}+\lambda_{6}^{D}$. A solution exists, if the term under the square root is positive, leading to the condition

$$
\lambda^{2} \geq 1-\left(\lambda_{\text {eff }}^{D} M_{0}\right)^{2} .
$$

This means that one can observe flow alignment in a ferromagnetic nematic, even if the pure nematic solvent shows tumbling behavior. 
We investigated the stability of the solution Eq. (72) numerically. We find that it is stable in the absence of a magnetic field, if the static coupling $\left(A_{1}\right)$ between the director and the magnetization is negative, because in that case $\mathbf{n} \perp \mathbf{M}$ is the equilibrium orientation. For a positive $A_{1}$, even the slightest perturbation in the magnetization field drives the director towards it, since it is favorable for them to be parallel rather than perpendicular. The solution Eq. (72) can nevertheless be made stable for ferromagnetic nematics with positive $A_{1}$, provided one uses a large magnetic field in $y$ direction and the diamagnetic anisotropy $\chi_{a}$ is negative. This is to ensure the director stays within the shear plane and the magnetization is perpendicular to it.

The case discussed above, where the magnetization is perpendicular to the shear plane and the director is in the shear plane at an angle $\theta$ with respect to the velocity field, most closely resembles one of the possible stationary solutions of a biaxial nematic liquid crystal exposed to a shear flow, see Ref. [43]. In the latter system the angle of one of the preferred directions with respect to the velocity field is given by the reversible coupling of the corresponding variable to the velocity field. In contrast, in ferromagnetic nematic liquid crystals this angle is determined by both, the reversible and the dissipative coupling of the director to the velocity field.

\section{SWITCH-ON DYNAMICS}

In this section we study the reorientation of the director and the magnetization in an external magnetic field applied perpendicularly to the glass plates, $\mathbf{H}=H \hat{\mathbf{e}}_{z}$. We are particularly interested in the influence of flow on this transient dynamics. Unlike in the previous sections, flow is not externally imposed, but is generated by the reorientation dynamics itself, i.e., by backflow. Throughout this section, the dissipative cross-coupling between the magnetization and the velocity $c_{i j k}^{D}$, Eq. (18), is put to zero.

As a first step we take into account only the reversible cross-coupling $\lambda_{i j k}$ between the director and the velocity, Eqs. (20)-(22), while the analogue cross-coupling $c_{i j k}^{R}$ between the magnetization and the velocity, Eqs. (19), (21) and (23), is put to zero. Fig. 9 shows the time dependence of $n_{z}$ in the mid-plane of the cell $\left(z=\frac{d}{2}\right)$. A comparison is made between cases with and without inclusion of flow. We find that the influence of the reversible director-flow coupling is small and makes the transient dynamics a little faster, see Fig. 9. This has also been readily encountered in usual nematic liquid crystals [28, 29]. We furthermore find that a strong dissipative cross-coupling between the director and the magnetization makes these backflow effects even less visible. In order to isolate them, we have put the tensor $\chi_{i j}^{D}$, Eq. (14), to zero.

When the term $c_{2}^{R}$ of the cross-coupling $c_{i j k}^{R}$, Eq. (23), is included in addition to $\lambda_{i j k}$, the backflow effects are similar, i.e., the dynamics is only slightly faster. This is



FIG. 9: The theoretical time dependence of $n_{z}$ in the middle of the cell, for the cases with and without inclusion of flow, $\mu_{0} H=5 \mathrm{mT}$. In the former case, only the flow alignment tensor $\lambda_{i j k}$ and the viscous tensor $\nu_{i j k l}^{D}$ are taken into account in the dynamics.

not surprising, since the tensor $\lambda_{i j k}$ and the term $c_{2}^{R}$ of have a similar form.

On the other hand we find that the dissipative crosscoupling between the director and the velocity $\lambda_{i j k}^{D}$, Eq. (17), which is absent in usual nematics, can have a somewhat larger influence, Fig. 10. In our numerical calculations we used the coefficient $\lambda_{3}^{D}$ as the representative of the tensor $\lambda_{i j k}^{D}$, i.e., $\lambda_{i j k}^{D}=\lambda_{3}^{D}\left(\epsilon_{i p k} M_{j} n_{p}+\epsilon_{i p j} M_{k} n_{p}\right)$.

The reason for this choice is that at $t=0$, when both $\mathbf{n}$ and $\mathbf{M}$ are parallel and within the $x z$ plane, $\lambda_{3}^{D}$ provides is a nonzero contribution to the director quasi-current component $Y_{y}$. The same is true for the coefficients $\lambda_{1}^{D}$ and $\lambda_{4}^{D}$, while the initial contributions of $\lambda_{2}^{D}, \lambda_{5}^{D}$ and $\lambda_{6}^{D}$ are zero. It should be noted that, due to the additional dissipative cross-coupling $\lambda_{i j k}^{D}$, the velocity field has a general orientation somewhere in the $x y$ plane and hence $\mathbf{n}$ and $\mathbf{M}$ wander out of the $x z$ plane, Fig. 10(b). This is in contrast to the case in Sec. III, where the velocity points along the $x$ direction throughout the cell and $\mathbf{n}$ and $\mathbf{M}$ stay in the shear plane.

The $v_{x}$ and $v_{y}$ profiles at four different moments are presented in Fig. 11 in terms of the Ericksen number, defined as the ratio of viscous and elastic forces on the director,

$$
\operatorname{Er}_{i}=\frac{\gamma_{1} v_{i} d}{K}
$$

with $i$ corresponding to either the $x$ or $y$ velocity component. For the chosen value of $\lambda_{3}^{D}$, the maximum values in both directions are comparable, $\operatorname{Er}_{x} \sim \operatorname{Er}_{y} \sim 2$.

It should be noted that the values of the dissipative cross-coupling coefficients are fundamentally restricted by the positivity of the entropy production. In our case (zero $\chi_{1}^{D}, \chi_{2}^{D}$ and $c^{D}$ ) the restriction is

$$
\left|\lambda_{3}^{D} M_{0}\right| \leq \sqrt{\frac{\nu_{3}}{\gamma_{1}}} \sim 0.75
$$



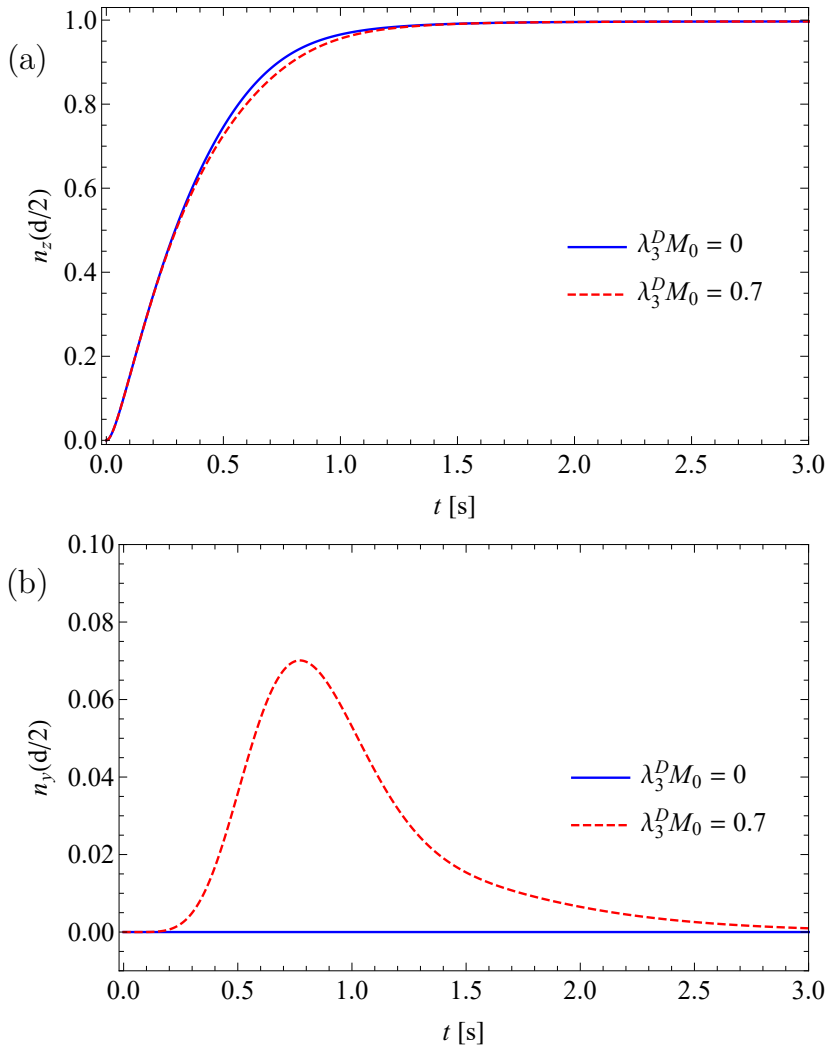

FIG. 10: The theoretical time dependence of the (a) $z$ and (b) $y$ component of the director field at $\mu_{0} H=5 \mathrm{mT}$ for two different values of $\lambda_{3}^{D}$.


FIG. 11: Backflow profiles in terms of the Ericksen numbers $\operatorname{Er}_{x}$ and $\operatorname{Er}_{y}$, Eq. (74), at four moments of time; $\mu_{0} H=5 \mathrm{mT}$, $\lambda_{3}^{D} M_{0}=0.7$.

where $\nu_{3}$ is chosen as the representative of the tensor $\nu_{i j k l}$.

We are interested in the importance of specific contributions to components $\sigma_{x z}$ and $\sigma_{y z}$ of the stress tensor. These are the usual nematic backflow-driving stress $\frac{1}{2} \lambda_{k i j} h_{k}^{n}$, the analogous stress corresponding to the dy-
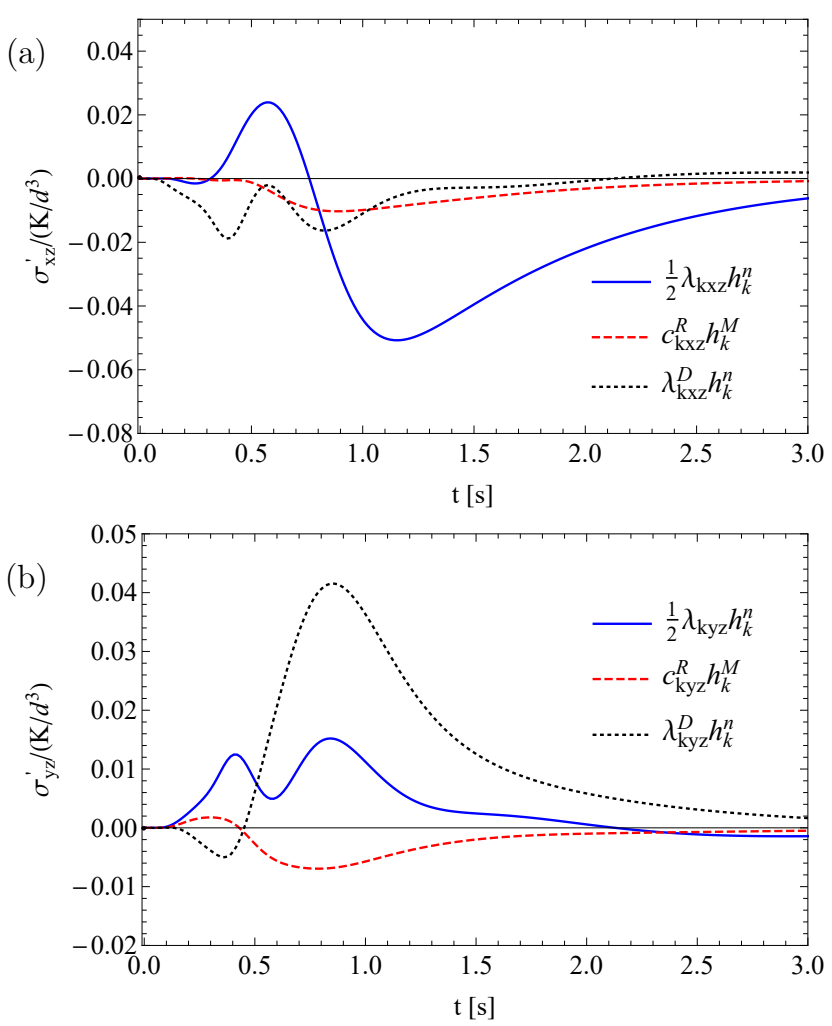

FIG. 12: The $x$ and $y$ components of the stress tensor divergence (a) $\sigma_{x z}^{\prime}=\partial \sigma_{x z} / \partial z$ and (b) $\sigma_{y z}^{\prime}=\partial \sigma_{y z} / \partial z$, at $z=d / 2$, in units of characteristic divergence of the elastic stress $K / d^{3}$ for each of the three different backflow-driving stress tensor contributions explained in the text.

namics of the magnetization, $c_{k i j}^{R} h_{k}^{n}$, and the stress from the dissipative director-velocity cross-coupling, $\lambda_{k i j}^{D} h_{k}^{n}$. The divergence of these stresses have non-zero $x$ and $y$ components that are individually presented in Fig. 12.

We first analyze the $\sigma_{x z}$ component, Fig. 12(a). The contributions of $c_{k x z}^{R} h_{k}^{n}$ and $\lambda_{k x z}^{D} h_{k}^{n}$ are smaller than those of $\frac{1}{2} \lambda_{k x z} h_{k}^{n}$. This can be explained by two facts. Firstly, at any moment the director field is much more deformed than the magnetization field, which means that the thermodynamic force $h_{i}^{n}$, Eq. (9), will have a much bigger impact on the divergence of the stress than the thermodynamic force $h_{i}^{M}$, Eq. (8). This automatically explains the backflow from the director field $\frac{1}{2} \lambda_{k x z} h_{k}^{n}$ is bigger than that from the magnetization $c_{k x z}^{R} h_{k}^{n}$. Secondly, the contribution $\lambda_{k x z}^{D} h_{k}^{n}$ is proportional to $\lambda_{3}^{D} n_{y} M_{z} h_{z}^{n}$. Since $n_{y}$ is never large, Fig. 10(b), so is the contribution of $\lambda_{k x z}^{D} h_{k}^{n}$.

The situation is different for the $\sigma_{y z}$ component. Fig. 12(b) reveals that the contribution of $\lambda_{k y z}^{D} h_{k}^{n}$ is the biggest. This can be explained by writing its leading term as $\lambda_{3}^{D} n_{x} M_{z} h_{z}^{n}$, which does not contain $n_{y}$. The leading terms of the backflow contributions, $\frac{1}{2} \lambda_{k y z} h_{k}^{n}$ and $c_{k y z}^{R} h_{k}^{n}$, are smaller in comparison, since they are always proportional to either $n_{y}$ and $M_{y}$, or to $h_{y}^{n}$ and $h_{y}^{M}$.

Next we are interested in the magnitude of the flow 
generated when a magnetic field is applied perpendicularly to the plates in one case and a voltage difference is applied across the plates in the other case. The magnitude of the flow is measured using the Ericksen number $\mathrm{Er}_{x}$, Eq. (74), which we have calculated using the maximum magnitude of the velocity across the cell. We find that $\operatorname{Er}_{x} \sim 1$ can be achieved using either a rather small magnetic field of $3 \mathrm{mT}$ or a voltage difference of 2.5 volts.

We conclude this section by pointing out that there are no effects of flow on the initial dynamics, at least up to first order in time. This can be shown by expanding the currents in Eqs. (3)-(5). Since the initial thermodynamic forces $h_{i}^{n}$ and $h_{i}^{M}$ are homogeneous, the generated stress $\sigma_{i j}$ in Eq. (5) is also homogeneous. Its divergence, which generates flow, is thus absent initially. Consequently Eqs. (3) and (5) are initially also unaffected by flow. It is the dissipative cross-coupling coefficient $\chi_{2}^{D}$ between the director and the magnetization that gives a linear time dependence of $n_{z}$, as was shown in Ref. [25] and discussed in Ref. [26].

\section{CONCLUSIONS AND PERSPECTIVE}

In this paper we have analyze the consequences of some simple flows on various configurations of ferromagnetic nematic liquid crystals. These flows include simple shear flow, the determination of transport coefficients à la Miesowicz and the analogue of flow alignment for ferromagnetic nematics.

For the case of simple shear we find that the effective viscosity can be increased by a factor of about two for rather small magnetic fields of about $20 \mathrm{mT}$. This effect can be tuned continuously in magnitude simply by varying the external magnetic field. For the determination of transport coefficients we analyze the analogue of the three Miesowicz configurations well-known from usual uniaxial nematics. Since one can fix the director and the direction of the spontaneous magnetization by external electric and magnetic fields, it is now possible to analyze nine independent different geometries. We find that these various geometries can be used to determine experimentally combinations of coefficients including dissipative and reversible terms characteristic of a system with a director, a magnetization and a velocity field as macroscopic variables.

For the analogue of flow alignment in usual uniaxial nematics we find simple stationary solutions without an external magnetic field involving an orientation of both, the director and the magnetization, for the case where both these variables are in the shear plane in the limit of sufficiently large shear. In addition, we show that a small external magnetic field can shift the boundary between tumbling and flow alignment. For the case that the magnetization $\mathbf{M}_{\mathbf{0}}$ is perpendicular to the shear plane and the director $\mathbf{n}$ is lying in the shear plane, we find for a range of parameters that a ferromagnetic nematic can reveal flow alignment although the nematic solvent by itself shows tumbling.

As a perspective we point out that we are aware of only one experimental publication investigating the effect of flow on ferromagnetic nematics, namely the effects of shear flow in a rather complicated geometry [35]. These experimental results are compatible with our theoretical results in the sense that they show qualitatively similar behavior as a function of an external magnetic field and a viscosity enhancement effect of comparable magnitude. In a next step it is highly desirable to compare experimental and theoretical results quantitatively for a simple and well-controlled geometry. There appear to be no experimental results available so far for the question of flow alignment and the evaluation of transport coefficients, parallel to the Miesowicz concept, for ferromagnetic nematics. Clearly, any experimental results in these two directions will stimulate refinement of the modeling of this exciting first liquid multiferroic system at room temperatures.

\section{Acknowledgments}

Partial support of this work through the Schwerpunktprogramm SPP 1681 'Feldgesteuerte Partikel-MatrixWechselwirkungen: Erzeugung, skalenübergreifende Modellierung und Anwendung magnetischer Hybridmaterialien' of the Deutsche Forschungsgemeinschaft is gratefully acknowledged, as well as the support of the Slovenian Research Agency, Grants J1-7435 and J1-7441 (D.S.).

\section{APPENDIX A: LESLIE COEFFICIENTS}

In the Ericksen-Leslie formulation of nematodynamics a different set of coefficients is used. The Leslie coefficients are defined [1] by the symmetrized stress tensor

$$
\begin{aligned}
\sigma_{i j}^{E L} & =\alpha_{1} n_{i} n_{j} n_{k} n_{p} A_{k p}+\left(\alpha_{2}+\alpha_{3}\right)\left(n_{i} N_{j}+n_{j} N_{i}\right) \\
& +\alpha_{4} A_{i j}+\left(\alpha_{5}+\alpha_{6}\right)\left(n_{i} n_{k} A_{j k}+n_{j} n_{k} A_{i k}\right),
\end{aligned}
$$

and the molecular field

$$
h_{i}^{E L}=\gamma_{1} N_{i}+\gamma_{2} n_{j} A_{i j}
$$

with $\gamma_{2}=\alpha_{3}+\alpha_{2}$ being a reversible transport parameter, not a viscosity. Here, the superscript $E L$ stands for Ericksen-Leslie, $N_{i}=\partial n_{i} / \partial t-\omega_{i j} n_{j}$ is the co-rotational time derivative of the director - or rather its dissipative quasi-current, $-Y_{i}^{D}$, Eq. (4), and $\omega_{i j}=\frac{1}{2}\left(\partial v_{i} / \partial x_{j}-\right.$ $\left.\partial v_{j} / \partial x_{i}\right)$, is the vorticity tensor.

The set of Leslie coefficients is related to the coeffi- 
cients of the tensor $\nu_{i j k l}^{D}[33]$ :

$$
\begin{aligned}
\alpha_{1} & =2\left(\nu_{1}+\nu_{2}-2 \nu_{3}\right)-\gamma_{1} \lambda^{2}, \\
\alpha_{2}+\alpha_{3} & =-\gamma_{1} \lambda, \\
\alpha_{4} & =2 \nu_{2}, \\
\alpha_{5}+\alpha_{6} & =4\left(\nu_{3}-\nu_{2}\right)+\gamma_{1} \lambda^{2} .
\end{aligned}
$$

The flow alignment parameter $\lambda=-\gamma_{2} / \gamma_{1}$ is expressed as the ratio of reversible transport parameter $\gamma_{2}$ and the rotational viscosity $\gamma_{1}$.
[1] P.G. de Gennes and J. Prost, The Physics of Liquid Crystals (Clarendon Press, Oxford, 1995).

[2] R.E. Rosensweig, Ferrohydrodynamics (Cambridge University Press, Cambridge, 1985).

[3] S. Odenbach, J. Phys. Condens. Matter 16, R1135 (2004).

[4] H.-W. Müller and M. Liu, Phys. Rev. E 64, 061405 (2001).

[5] B. Huke and M. Lücke, Rep. Prog. Phys. 67, 1731 (2004).

[6] P. Ilg, E. Coquelle, and S. Hess, J. Phys. Condens. Matter 18, S2757 (2006).

[7] S. Mahle, P. Ilg, and M. Liu, Phys. Rev. E 77, 016305 (2008).

[8] S.D. Peroukidis and S.H.L. Klapp, Phys. Rev. E 92, 010501 (2015).

[9] F. Brochard and P.G. de Gennes, J. Phys. (France) 31, 691 (1970).

[10] A. Mertelj, D. Lisjak, M. Drofenik, and M. Čopič, Nature 504, 237 (2013).

[11] A. Mertelj and D. Lisjak, Liq. Cryst. Rev. 5, 1 (2017).

[12] A.J. Leggett, Rev. Mod. Phys. 47, 331 (1975).

[13] J.C. Wheatley, Rev. Mod. Phys. 47, 415 (1975).

[14] R. Graham, Phys. Rev. Lett. 33, 1431 (1974).

[15] R. Graham and H. Pleiner, Phys. Rev. Lett. 34, 792 (1975).

[16] M. Liu, Phys. Rev. Lett. 43, 1740 (1979).

[17] H. Brand, M. Dorfle, and R. Graham, Ann. Phys. (N.Y.) 119, 434 (1979).

[18] H. Brand and H. Pleiner, J. Phys. (France) 43, 369 (1982).

[19] Q. Zhang, P.J. Ackerman, Q. Liu, and I.I. Smalyukh, Phys. Rev. Lett. 115, 097802 (2015).

[20] P.J. Ackerman and I.I. Smalyukh, Nature Materials 16, 426 (2017).

[21] P. Medle Rupnik, D. Lisjak, M. Čopič, S. Čopar, and A. Mertelj, Sci. Adv. 3, e1701336 (2017).

[22] H.R. Brand, A. Fink, and H. Pleiner, Eur. Phys. J. E 38, 65 (2015).

[23] A. Mertelj, N. Osterman, D. Lisjak, and M. Čopič, Soft Matter 10, 9065 (2014).

[24] H. Pleiner, E. Jarkova, H.-W. Müller, and H.R. Brand, Magnetohydrodynamics 37, 254 (2001).
[25] T. Potisk, D. Svenšek, H.R. Brand, H. Pleiner, D. Lisjak, N. Osterman, and A. Mertelj, Phys. Rev. Lett. 119, 097802 (2017).

[26] T. Potisk, A. Mertelj, N. Sebastián, N. Osterman, D. Lisjak, H.R. Brand, H. Pleiner, and D. Svenšek, Phys. Rev. E 97, 012701 (2018).

[27] D. Forster, Hydrodynamic Fluctuations, Broken Symmetries and Correlation Functions (W.A. Benjamin, Reading, Mass., 1995).

[28] D. Svenšek and S. Žumer, Liq. Cryst. 28, 1389 (2001).

[29] D. Svenšek, S. Žumer, Continuum Mech. Thermodyn. 14, 231 (2002).

[30] J. Turk and D. Svenšek, Phys. Rev. E 89, 032508 (2014).

[31] E. Jarkova, H. Pleiner, H.-W. Müller, A. Fink, and H.R. Brand, Eur. Phys. J. E 5, 583 (2001).

[32] E. Jarkova, H. Pleiner, H.-W. Müller, and H.R. Brand, J. Chem. Phys. 118, 2422 (2003).

[33] H. Pleiner and H.R. Brand, Hydrodynamics and Electrohydrodynamics of Nematic Liquid Crystals, in Pattern Formation in Liquid Crystals, edited by A. Buka and L. Kramer (Springer, New York, 1996).

[34] L.M. Blinov and V.G. Chigrinov, Electrooptic Effects in Liquid Crystal Materials (Springer, New York, 1996)

[35] R. Sahoo, M.V. Rasna, D. Lisjak, A. Mertelj, and S. Dahra, Appl. Phys. Lett. 106, 161905 (2015).

[36] D. Collin, G.K. Auernhammer, O. Gavat, P. Martinoty, and H.R. Brand, Macromol. Rapid Commun. 24, 737 (2003).

[37] S. Bohlius, H.R. Brand, and H. Pleiner, Phys. Rev. E 70, 061411 (2004).

[38] M. Miesowicz, Nature 136, 261 (1935).

[39] M. Miesowicz, Nature 158, 27 (1946).

[40] H. Brand and H. Pleiner, Phys. Rev. A 24, 2777 (1981).

[41] M. Liu, Phys. Rev. A 24, 2720 (1981).

[42] A. Saupe, J. Chem. Phys. 75, 5118 (1981).

[43] H. Brand and H. Pleiner, J. Phys. 43, 853 (1982).

[44] H. Pleiner and H.R. Brand, J. Phys. 46, 615 (1985).

[45] K. Skarp and T. Carlsson, Mol. Cryst. Liq. Cryst. 49, 75 (1978).

[46] T. Carlsson and K. Skarp, Mol. Cryst. Liq. Cryst. 78, 157 (1981). 